\title{
Homosexualidad rural en los Andes: notas desde los Yungas de La Paz, Bolivia
}

Homosexualité rurale dans les Andes : notes depuis la région des Yungas de La Paz, Bolivie

Rural Andean homosexuality: fieldnotes from the Yungas of La Paz, Bolivia

\section{Alison Spedding y Helan Vichevich}

\section{(2) OpenEdition}

Journals

Edición electrónica

URL: http://journals.openedition.org/bifea/8084

DOI: $10.4000 /$ bifea.8084

ISSN: 2076-5827

Editor

Institut Français d'Études Andines

Edición impresa

Fecha de publicación: 1 diciembre 2016

Paginación: 433-450

ISSN: 0303-7495

Referencia electrónica

Alison Spedding y Helan Vichevich, « Homosexualidad rural en los Andes: notas desde los Yungas de La Paz, Bolivia », Bulletin de l'Institut français d'études andines [En línea], 45 (3) | 2016, Publicado el 08 diciembre 2016, consultado el 05 noviembre 2020. URL : http://journals.openedition.org/bifea/8084 ; DOI : https://doi.org/10.4000/bifea.8084

\section{(c)}

Les contenus du Bulletin de l'Institut français d'études andines sont mis à disposition selon les termes de la licence Creative Commons Attribution - Pas d'Utilisation Commerciale - Pas de Modification 4.0 International. 


\title{
Homosexualidad rural en los Andes: notas desde los Yungas de La Paz, Bolivia
}

\author{
Alison Spedding ${ }^{*}$ con Helan Vichevich ${ }^{* *}$
}

\begin{abstract}
Resumen
La poca bibliografía existente sobre homosexualidad en los países andinos trata casi exclusivamente de contextos urbanos. En cuanto al ámbito rural, considerado por definición «indígena», muchos activistas políticos de corriente indianista insisten en que la homosexualidad no existe en la cultura andina, siendo una perversión introducida por la colonia española; si se logra identificar a una persona como homosexual en una comunidad andina, este individuo sería rechazado tajantemente. Ambos casos enfocan la homosexualidad masculina. Este ensayo presenta estudios de caso de homosexualidad tanto masculina como femenina en dos provincias bolivianas, demostrando que estas sexualidades son reconocidas en la cultura local y que las personas que las exhiben participan en actividades públicas y privadas sin ser objetos de discriminación ni rechazo.
\end{abstract}

Palabras clave: homosexualidad, andina, rural, Bolivia, sexualidad

\section{Homosexualité rurale dans les Andes : notes depuis la région des Yungas de La Paz, Bolivie}

\section{Résumé}

Le peu de littérature existante sur I'homosexualité dans les pays andins traite presque exclusivement des contextes urbains. En ce qui concerne l'espace rural, considéré par définition comme «indigène», beaucoup d'activistes politiques appartenant au courant indianiste persistent à dire que l'homosexualité n'existe pas dans la culture andine, qu'il s'agit d'une perversion introduite par la colonisation

* Carrera de Sociología, Universidad Mayor de San Andrés, La Paz, segundo piso, Edificio Rene Zavaleta Mercado, Monoblock Central, Avenida Villazón s/n, La Paz. E-mail: mamahuaco@hotmail. co.uk

** Estudiante de la Universidad Mayor de San Andrés, La Paz, Bolivia. 
espagnole et, qu'en cas d'identification d'une personne comme homosexuelle dans une communauté andine, celle-ci serait rejetée catégoriquement. Dans les deux cas, les discours se concentrent sur I'homosexualité masculine. Cet article présente des pratiques d'homosexualité masculine et féminine observées dans deux provinces rurales boliviennes, montrant que ces sexualités sont reconnues dans la culture locale et que les gens qui les affichent participent à des activités publiques et privées sans être victimes de discrimination ou de rejet.

Mots-clés : homosexualité, andine, rural, Bolivia, sexualité

\title{
Rural Andean homosexuality: fieldnotes from the Yungas of La Paz, Bolivia
}

\begin{abstract}
The scarce literature on homosexuality in Andean countries almost exclusively treats urban contexts. With reference to rural settings, considered "indigenous" by definition, many political activists with Indianist postures insist that homosexuality does not exist in Andean culture, being a perversion introduced by Spanish colonization. If homosexual persons were identified in an Andean community, they would be totally rejected. In both cases the focus is on male homosexuality. This essay presents case studies of both male and female homosexuality in two Bolivian provinces, demonstrating that these sexualities are recognized in the local culture and that the people who display them participate in public and private activities without being subject to discrimination or rejection.
\end{abstract}

Keywords: homosexuality, Andean, rural, Bolivia, sexuality

Iniciaremos este artículo con una cita extensa del antropólogo gibraltareño Andrew Canessa, que ha realizado un trabajo de campo prolongado y en idioma nativo (aymara) en una comunidad de la provincia Larecaja, en los valles del departamento de La Paz. Procede de una nota de pie respecto a las películas pornográficas que los hombres de esta comunidad ven cuando van a trabajar en las minas de oro en la parte baja (selvática) de esta misma provincia, en particular las de «sexo lésbico»:

«Sexo lésbico» quizás es una descripción equivocada: los wilakjarkeños con quienes hablé no asociaban las escenas de dos mujeres teniendo relaciones sexuales en un video con lo que se podría considerar como «lesbianas». Quizás con razón tratando de pornografía, interpretaban tales actos sexuales como carentes de relación con algún tipo de vínculo de pareja entre las mujeres involucradas, o una sexualidad particular; de hecho, la gente asombraba al considerar que podría existir tal cosa. Me dijeron que las mujeres no podrían tener relaciones sexuales (con penetración) ni podrían formar una pareja, porque para dirigir una unidad doméstica se requiere un hombre y una mujer. Por supuesto, no hay una palabra para «lesbiana» en Wila Kjarka; hay una palabra para «marimacho», mujer que actúa como un hombre, urquchi, pero no refiere a su sexualidad. De manera parecida, no hay una palabra para un hombre homosexual. 
Q'iwsa, que aparentemente significa un varón homosexual en otras partes de los Andes, es usado en Wila Kjarka para referir a un hombre mezquino, que no cumple con sus obligaciones sociales y no bebe «como es debido» (es decir, en gran cantidad) en las fiestas. Sí se utiliza la palabra castellana «maricón» para referir a hombres mestizos que tienen relaciones con otros hombres. Los hombres en Wila Kjarka hablan de «maricones» con miedo, sorna y revulsión (Canessa, 2006: 135-136).

Esta cita destaca elementos que, como veremos, son centrales en las concepciones de la homosexualidad en las comunidades rurales: el acto de penetración como núcleo de una relación sexual; la diferenciación de la homosexualidad femenina de la masculina; la importancia de conformar una unidad doméstica como sinónimo de la vida en pareja; además una actitud de rechazo hacia los homosexuales masculinos. La referencia a que son los hombres mestizos que «tienen relaciones con otros hombres» es conforme con lo que Silvia Rivera, en una entrevista publicada en 2013, reportó de un joven indianista quien le indicó que «el homosexualismo es una enfermedad traída por los españoles». Ella misma opina que tal posición se debe a la influencia en el indianismo de «gente letrada con ideas muy inventadas de lo que pudo haber sido una tradición» y no es propia de «las bases de la comunidad» que a su parecer tendrián «mayor tolerancia» (Rivera, 2013: 27). Canessa me confirmó, en una conversación personal, que en la comunidad donde él trabaja no había ningún caso de homosexualidad conocida, y que a su parecer la gente desconocía tal condición. La oración final de la cita indica que, en tanto habrían tenido algún conocimiento de relaciones homosexuales —exclusivamente masculinas-, se trataba de algo que ocurría entre personas totalmente ajenas a su propio grupo social y por tanto, igualmente ajeno a ellos mismos. Una posición similar es expresada en los escritos de Edson Hurtado, activista gay y periodista, que ha publicado una colección de cuentos y aforismos (Hurtado, 2011) y «crónicas» (estas últimas basadas en un viaje «de seis meses a más de una docena de pueblos indígena-originarios», Hurtado, 2015). Tratándose de mineros potosinos, de guaranís en Santa Cruz o de una madre campesina en un zona rural no especificada, lo común es el rechazo y la expulsión de la comunidad, cuando no el castigo físico brutal que puede llegar hasta el asesinato.

Hasta aquí, entonces, parecería que los indianistas, letrados o no, tendrían razón en aseverar que la homosexualidad es ajena a las comunidades andinas. Pero contrasta con la posición de Alejandro Ortiz Rescaniere. Este autor no precisa a qué regiones o comunidades se refiere, pero se deduce de su texto que el contexto general es la sierra sur quechuahablante del Perú. Sobre el tema de «amar a uno del mismo sexo», dice:

En principio no es mal visto, por el contrario ... Cuando dos amigos ... tienen algunas actitudes o comportamientos de dos amantes las peores censuras que recibirán serán unas bromas... Si el par de amigos se propasara de estos límites y llegara a tener relaciones más bien físicas, éstas no acarrean mayores rumores ni menos consecuencias cósmicas o monstruosas. La reprobación, si la hay, será mínima. ... A veces hemos escuchado que en tal pueblo o en la época de los bisabuelos había una 
pareja de dos personas del mismo sexo (sobre todo, de dos mujeres). Una se comportaba como si fuese del otro sexo. Estas anécdotas se cuentan con cierta inquietud. Son percibidas como rarezas de la naturaleza («Así nacieron.») (Ortiz Rescaniere, 1993: 105-106).

El autor no profundiza el tema con datos etnográficos, suyos o de otros investigadores, sino que procede a comentar alguna referencia a la homosexualidad ritual prehispánica, el travestismo ritual actual en los bailes, y cierto valor positivo, en el contexto de la adivinación en coca, que se puede atribuir al ch'ulla (cosa suelta que no tiene su pareja), sugiriendo que tales cosas pueden llega a ser illa, cosa única, que no falta tener pareja y a la vez es un amuleto, talismán y fuente de fertilidad. No voy a ocuparme de estos temas históricos y simbólicos —que también son comentados por Rivera (2013) — sino recuperar las conclusiones del acápite del texto de Ortiz referente a la vida social actual:

La actitud andina hacia la homosexualidad... cuando se presenta de manera episódica, en la vida corriente, no se le presta mayor atención (Ortiz Rescaniere, 1993: 107).

Sin embargo, me parece excesivamente especulativa su propuesta de que:

Una relación homosexual prolongada o estable quizás hasta sea percibida por los vecinos como benéfica (podría ser bueno tener una illa cerca) (Ortiz Rescaniere, 1993: 107-108).

El travestismo en los bailes tiene interés para analizar el simbolismo del género, pero no tiene relación con la sexualidad de los (y con mucho menor frecuencia, las) que se travisten excepto, como me dijo un informante, cuando cierto hombre «insiste en hacerlo todas las veces» (Teodoro Mamani, El Alto, 1996). Hasta la década de los años 1980, el baile de la morenada en la ciudad de La Paz era de varones, y las primeras «chinas morenas» - figuras con una versión paródica de la vestimenta de la chola paceña ${ }^{1}$ donde se destaca una pollera convertida en minifalda en vez de las convencionales que, hasta ahora, llegan recatadamente hasta media pantorilla-, eran precisamente varones homosexuales travestidos. Activistas gay como el grupo «Familia Galan» recuperaron y publicitaron esta tradición en los años 2000 (cf. el texo de David Aruquipa en este mismo número). En tanto que se generalizó la figura de las chinas, eran asumidas por mujeres biológicas. Lo que se podría llamar travestismo ritual en los bailes que hoy en día se acostumbra llamar «autóctonos» —de tradición localista, con disfraces confeccionados por los participantes y no alquilados de bordadores urbanos comerciales- es, en el área rural, asumido por varones convencionalmente heterosexuales, de manera jocosa y sin valor ritual consciente.

Obviando los casos de parejas establecidas del mismo sexo, se supone que los homosexuales — como se verá, aquí utilizo intencionalmente el masculino- van a vivir solos, es decir, si no viven con sus padres o - tal vez- asimilados a la unidad doméstica de algún pariente colateral, conforman una unidad doméstica

1 Se refiere a la vestimenta de las mujeres rurales y de clase baja y media baja urbana, consistiendo en una pollera (falda amplia y plisada con bastas) colocada encima de varias enaguas, blusa, chompa abierta, manta y sombrero bombín. 
unipersonal y, en todo caso, son solterones. Otro de los clichés de la antropología andina es que se exige formar parte de una pareja conyugal, marido y mujer, en aymara chachawarmi, para asumir cargos en la comunidad, y a la vez es obligatorio hacer estos cargos para legitimar la posesión de la tierra. Esto es más bien la expresión de una realidad empírica: uno empieza a pasar cargos (o la gente empieza a exigir que uno los haga) cuando establece una unidad doméstica propia; para la vasta mayoría de la gente, esto ocurre después de haberse juntado con una persona del sexo opuesto apartándose de las unidades domésticas de sus respectivos padres. Las personas solteras, o mejor dicho solterones, también serán obligadas a cumplirlos, pero a mayor edad (digamos, por encima de los 30 años en vez de apenas llegando a los 20) cuando es evidente para todos los que poseen terrenos que manejan de forma independiente y no dentro de la economía común de su unidad doméstica de origen. «Servir a la comunidad», es decir gastando tiempo y recursos en pasar cargos de autoridad, es necesario para garantizar el derecho a la tierra. Max, entrevistado en Vargas \& Aruquipa (2013), asevera que en su comunidad en el Altiplano paceño, los homosexuales no pueden llegar a cargos porque «son bien machistas las autoridades» (Max in Vargas \& Aruquipa, 2013: 9), y que corrieron rumores que, por ser homosexual, la comunidad le iba a quitar su terreno.

Otro entrevistado, o más bien entrevistada, que figura en la misma publicación con su nombre «trans» de Antonella, expresa que a ella sí le obligaron a hacer cargo en su comunidad, y hubiera sido un beneficio ser librado del mismo por ser homosexual (Vargas \& Aruquipa, 2013: 19). La misma indica que un tío suyo, solterón pero no homosexual según lo que se sabe, había hecho varios cargos en la comunidad. Silvia Rivera considera que sí hay la exigencia de presentarse con una pareja de sexo opuesto para asumir un cargo de dirigente, así que los hombres sin cónyuge deben actuar «acompañados en todos los eventos por la hermana, por la tía o la mamá ... tiene que haber una figura simbólica de hombre y mujer» (Rivera, 2013: 30). «Todos los eventos» refiere propiamente a todos los eventos protocolares, como el juramento al cargo, fiestas o ritos, donde se puede exigir que la autoridad actúe en pareja; no son requisitos para el ejercicio de las tareas regulares de la dirigencia, como dirigir reuniones, asistir a asambleas, representar la comunidad y presentar solicitudes ante la alcaldía o un ministerio, etc.. En todo caso, es algo más típico del Altiplano u otras regiones donde hay un mayor componente ritual en el ejercicio de la autoridad comunal. Por lo tanto, no tener esposa no es en sí un obstáculo para ser autoridad, y menos algo que pone en riesgo la misma posesión de la tierra, siempre que la persona no busca tener conflictos con la comunidad por otros motivos; entre estos su sexualidad en sí no suele desempeñarse como causa.

\section{EL CASO ESTUDIADO: ALGO ACERCA DE LOS YUNGAS}

El resto de este artículo trata de casos reales de homosexualidad masculina y femenina que hemos encontrado en comunidades de los Yungas del departamento 
de La Paz en Bolivia. Los Yungas son los valles subtropicales, en particular los pisos ecológicos entre 2000 y 600 msnm, al norte de la ciudad de La Paz, pasando la cordillera oriental. Es una zona bastante extensa; en este texto, se va a hacer referencia a las zonas tradicionales (originalmente prehispánicas) de producción de la hoja de coca en las provincias de Sud Yungas e Inquisivi, donde Spedding ha estado haciendo trabajo de campo a partir de 1987. Hasta 1953 la zona estaba mayormente ocupada por haciendas, y a partir de entonces se ha organizado en sindicatos agrarios. Casi todas las comunidades yungueñas tienen servicios de agua potable por cañería, luz eléctrica y acceso por camino carretero. Los ingresos de la coca han permitido a la casi totalidad de los pobladores adquirir teléfonos celulares, aparatos electrodomésticos y en un buen porcentaje, automóviles particulares «chutos» (ingresados ilegalmente al país por contrabando desde Chile) y hasta antenas para recibir canales televisivos por satélite. La totalidad de los habitantes hablan castellano; en el campo, los niños y jóvenes son bilingües pasivos en aymara y su práctica de este idioma se vuelve activa en la adultez, llegando en casos a la pasividad en castellano en la ancianidad. La vestimenta tradicional de la región desapareció ya por los años 1940; la mayoría de las mujeres campesinas adultas aún visten la pollera, en los estilos corrientes de las clases bajas y media bajas urbanas de las ciudades de La Paz y El Alto (donde también poseen con frecuencia una segunda residencia) pero también ya hay madres de familia adultas que visten pantalón, lo que no era el caso cuando Spedding hizo su primer trabajo de campo en 1986-1988. Ni ahora ni entonces los y las dirigentes sindicales (hay una organización de mujeres afiliada a «las Bartolinas») utilizan distintivo alguno como es habitual en el Altiplano cuando acuden a congresos o marchas campesinas en la ciudad de La Paz. Suelen decir de las delegaciones altiplánicas cuyos miembros llevan todos ponchos, polleras, chalinas, aguayos 2 y chicotes de determinados colores y modelos, «Uniformados han venido». Ellos simplemente vienen con su ropa cotidiana y no hay manera de saber quiénes son los dirigentes principales y quiénes son subalternos o bases si uno no les conoce personalmente. Todo esto conduce a una impresión difundida que «los Yungas son aculturados», reacción escuchada por Spedding en diversas ponencias suyas donde cuestionaba versiones a su parecer excesivamente esencializadas e idealizadas del «mundo andino». ¿Será que sus actitudes relajadas hacia la homosexualidad son también una expresión de aculturación?

\section{HOMOSEXUALIDAD MASCULINA}

Este estudio se inició con un trabajo que Vichevich hizo en 2013 para una de las materias que cursaba en la Carrera de Sociología de la Universidad Mayor de San Andrés, en la comunidad de Takipata ${ }^{3}$ cerca a Chulumani, la capital de la

2 Nombre aymara que designa una tela grande y cuadrada, generalmente con un diseño de listas y figuras de colores vistosos, que las mujeres utilizan para cargar a niños y a toda clase de objetos en sus espaldas

3 Todos los nombres de comunidades y personas son apodos o seudónimos. 
provincia de Sud Yungas. Pudo ingresar con relativa facilidad a este tema que no se considera de incumbencia pública — como indicó un entrevistado, «no importa lo que haga la gente en sus casas... hay que dejar en paz al Gordo» (apodo de un homosexual conocido de la comunidad), es decir, no «molestarle» sacando detalles de su vida privada a la luz - porque ya había visitado la comunidad varias veces en años anteriores en compañía de Spedding quien es afiliada de la misma. Por tanto Spedding ya le informó quiénes eran homosexuales y Vichevich no tuvo que escarbar para identificarles, sino directamente indagar sobre las actitudes que la gente tenía hacia ellos. En primer lugar, encontró que no hay obstáculos para que ellos sean dirigentes. Vale la pena mencionar que en el sindicalismo yungueño no se exige la presencia de la esposa (o del esposo, tratándose de dirigentes mujeres) ni siquiera en actos protocolares, así que no ser casado no afecta el ejercicio del cargo. El Gordo es afiliado de la comunidad y había ocupado el cargo de Presidente Comunal de ADEPCOCA4 . Posteriormente ha ocupado el cargo de Secretario de Medioambiente.

El otro homosexual conocido, Bernabé, no es afiliado pero había cumplido el cargo de Secretario Vocal en lugar de su padre, ya anciano y además sordo. Asumió como preste 5 de la fiesta patronal de la comunidad, la de la Virgen de la Concepción, el 8 de diciembre de 2015. Esto no es un hecho único. En 1989, el cargo menor de altarero (encargado de adornar la iglesia, y en la víspera de la fiesta ofrecer servicio de ponche) fue asumido por Salomón, un homosexual notorio que regentaba una pensión en el pueblo y era devoto de la Virgen aunque no tenía otro vínculo particular con Takipata. Es cierto que, en opinión de los asistentes, tanto la comida servida como el grupo musical proporcionado por Bernabé no eran de la mejor calidad comparado con años pasados; esto fue atribuido a que no tenía muchos recursos económicos y no fue relacionado con su sexualidad.

De hecho, a diferencia del Gordo, desde varios años Bernabé no reside en la comunidad misma, sino en el pueblo de Chulumani (durante una temporada, incluso vivió en el pueblo nuevo de Pastopata en otro distrito del municipio) donde ha regentado en diferentes periodos una tienda de abarrotes, un puesto de salchipapas y una pequeña pensión. Se apartó de la casa de sus padres por decisión personal, no por conflictos relacionados con su sexualidad, sino porque su padre durante años ha pegado a su madre hasta provocarle la inhabilitación permanente de uno de sus brazos. Junto con otro de sus hermanos que tampoco vive en Takipata porque es marido uxorilocal en otra comunidad colindante con el pueblo de Chulumani, Bernabé encabezó una demanda policial en contra de su padre por estos abusos, lo que provocó su alejamiento del hogar parental. Es por este motivo que hasta la fecha tampoco goza de terrenos que su padre le haya

4 Su principal obligación es emitir el Orden Comunal, un formulario que indica la cantidad y lugar de origen de la hoja de coca a ser llevado al Mercado Legal de la Coca en la ciudad de La Paz, para fines del control gubernamental el cual busca evitar que la hoja sea «desviada» hacia el narcotráfico.

5 Cargo que corresponde a contratar al cura para que de la misa de fiesta, pagar a músicos para amenizar el baile y servir comida a todos los asistentes, junto con las bebidas alcohólicas que quisiera proporcionar. 
asignado en herencia. El Gordo, en contraste, procede de una de las familias más acomodadas de la comunidad. Siendo el menor de los hijos que han quedado en la comunidad, ha heredado la amplia casa paternal, otra casa en el centro de la comunidad y ha juntado las parcelas que le correspondieron en herencia, otras compradas o cedidas por hermanos/as (incluyendo a una hermana adoptiva, en realidad nieta) que ya no viven en la comunidad y no les interesa manejar sus terrenos, algo comprensible ya que se exige cumplir con los deberes de un afiliado al sindicato para mantener el derecho propietario. En consecuencia, cuando la comunidad entró en el proceso legal de saneamiento de tierras a partir del año 2012, llegó a registrar diez parcelas, mientras la vasta mayoría registró entre dos y cuatro. Otra muestra de su nivel económico superior al promedio es que es una de las dos personas en la comunidad que poseen una computadora personal.

Estos dos hombres viven formalmente como solterones y sin parejas reconocidas, aunque en el pasado se supo de una relación estable entre el Gordo y un hombre de una comunidad originaria cercana. La dificultad de manejar solo una unidad doméstica campesina, es decir cubrir tanto el trabajo doméstico (más que todo, cocinar) como las labores agrícolas — que en los Yungas son todo el año, no hay una época muerta como en el Altiplano- es reconocida como una desventaja de ser q'iwsa, palabra aymara para hombre homosexual. Se dice «todo maricón sabe cocinar». Tanto el Gordo como Bernabé se han dedicado por temporadas a la venta de comida cocida, y a veces otras familias ruegan al Gordo para que les preste ayuda preparando la comida para algún rito de crisis vital, por ejemplo un entierro. Dentro de la división de trabajo por género, la tarea agrícola emblemática de la mujer es la cosecha de coca, que además es la etapa productiva que demanda mayor inversión de mano de obra. En una pareja heterosexual, es la esposa quien se ocupa de reclutar cosechadoras, sea en ayni (intercambio de jornadas laborales) o como mingas (con jornal pagado en dinero) mientras el marido es liberado de cosechar para dedicarse al desyerbe, la plantación de cocales nuevos y demás exigencias. Un solterón o un viudo necesariamente tiene que involucrarse en la cosecha.

En algún momento, el Gordo llegó a ser «contratista» de un grupo de unas doce o quince cosechadoras (básicamente, las que caben en un minibús), quienes se ofrecen a ir a trabajar a jornal para cualquier persona que les contrate. El o la contratista va a cosechar junto con ellas y recibe otro jornal adicional en reconocimiento de sus servicios. Aparte de su función productiva, la cosecha de coca (o k'ichi), siendo altamente socializada, es reconocida como un modo de comunicación muy efectivo, fuente constante de noticias, rumores y chismes de todo tipo. Estando en contacto permanente con numerosas mujeres, el Gordo participa en esto: según un informante, «todo lo que ve habla nomás. Como es maricón se mete nomás con mujeres y hombres, por eso habla mucho», expresando así una conducta considerada femenina (aunque los maridos dependen de sus mujeres para enterarse ellos mismos de las últimas noticias locales, ya que los trabajos masculinos se realizan en gran parte de manera solitaria, solo ocasionalmente en grupos que sobrepasan dos o tres miembros). Aparte de eso, ni él ni Bernabé ostentan conductas afeminadas (en sus gestos, en su vestimenta...), aunque eso 
sí era el caso de Salomón, de quien incluso se decía que alguna vez, al ir a su pensión, solo encontraron a «una cholita» en la cocina, que resultaba ser el mismo Salomón travestido. Tan pronunciado era su afeminamiento que, cuando estaba vendiendo cerveza en la fiesta de la comunidad de Tulduchi en 1991, una mujer que se acercó a comprarle se dirigió a él sin pensar como «señora», lapsus que fue festejado a carcajadas tanto por el referido como por ella y las demás personas presentes.

Tanto Bernabé como el Gordo han bailado en comparsas folklóricos, siempre como varones con los disfraces correspondientes y nunca como figuras travestidas. En 2011, Takipata presentó el baile tradicional de Loco Palla Palla en la entrada autóctona de la fiesta del pueblo en ese año. Este baile siempre incluye al menos uno o dos hombres vestidos como mujeres campesinas, con pollera y muchas veces una guagua cargada en aguayo en sus espaldas, denominadas rabonas (las esposas o compañeras de los soldados que en el siglo XIX iban a la guerra junto con ellos). Esto es un baile burlesco y las rabonas actúan de una manera deliberadamente provocativa, alzando sus polleras. Una comunaria criticó a los jóvenes que hacían este papel en 2011 porque levantaban sus polleras y enaguas por delante, indicando que esto «estaba mal» (pasaba de ser burlesco hasta rayar con lo obsceno), porque lo correcto era elevar sus faldas por atrás, es decir, ofreciendo sus nalgas, y no, si fueran mujeres biológicas, su vulva (y en la realidad, su pene). Es posible ver este gesto como una invitación homosexual velada (y tal vez por eso no hemos observado a hombres realmente homosexuales en este papel, porque en su caso iría más allá de un ofrecimiento meramente burlesco), pero en opinión de Spedding más bien apunta a subrayar que no son realmente mujeres y su aparente disponibilidad promiscua es exactamente eso, una burla sin relación con la realidad.

El Gordo no solo reúne a mujeres para la cosecha, sino también suele reunir a grupos de hombres jóvenes, mayormente solteros, para que hagan el desyerbe de sus cocales. En una ocasión, al volver del trabajo, se burlaron de él en grupo bajándole el pantalón. Los comentarios al respecto no indicaron que habrían procedido a abusar sexualmente de él, aunque otro informante indica que suele tener relaciones con sus jornaleros varones. El «abuso» que sí cometieron era reunirse con él en la tienda que regentaba durante unos años en el centro de la comunidad, invitarle a tomar cerveza con ellos, y cuando estaba borracho aprovechar para llevarse más bebida sin pagar. Las pérdidas ocasionadas le convencieron de cerrar la tienda. Lo notable de este episodio, al menos para Spedding, era que la gente criticó a estos jóvenes y se compadeció del Gordo; nadie sugirió que él tenía la culpa por «llamarles», y mucho menos que tal conducta podría conducir a la «corrupción» de los jóvenes, induciéndoles a la homosexualidad. En parte, esto puede reflejar la idea generalizada en los Andes (y otras partes del mundo) de que, cuando dos varones tienen relaciones sexuales anales, solo el penetrado es «homosexual» $y$ no el que penetra. En el curso de una borrachera, un hombre casado de Takipata 
contó a Vichevich que en otra sesión de borrachera en una casa particular, el Gordo empezó a tocarle los testículos y salieron afuera a internarse en la maleza con intenciones de tener relaciones sexuales. Solo que, según él, al bajarle los pantalones se sorprendió al descubrir que «no tenía chupila» (vulva en aymara) porque creía que «los maricones tienen los dos», es decir, que son hermafroditas. Decepcionado, se retiró sin ir a más, aunque no sin haber constatado que el Gordo era «ch'ulla huevo» (con un solo testículo), que consideró podría haber sido la causa de su homosexualidad. No hemos podido comprobar estas referencias, ni a la creencia ni a la anatomía, con otros informantes. Tal vez correspondan más bien a una metáfora de la situación del homosexual en cuanto a la división sexual del trabajo: tiene que hacer tanto de hombre como de mujer. Es casi inconcebible que se pueda vivir exclusivamente entre personas de un mismo sexo.

Al preguntarles si conocían castigos para los homosexuales, todos los informantes de Takipata coincidieron en que en los Yungas no los hay ni debe haber, «no se puede castigar a las personas por lo que hacen en sus casas si no perjudican», y que si hubiera lugares en el Altiplano donde esto ocurriera, sería porque allá «son ignorantes». Uno de los informantes indicó que, personalmente, él consideraba que la homosexualidad (entre varones, no mencionó el caso de las mujeres) es algo malo que merece ser prohibido, y que la comunidad no debería dejar a dos hombres vivir juntos, aunque otros pensaban que no serían rechazados. Pero es un punto especulativo, porque no se conoce caso real alguno donde lo hayan intentado. Otro señaló que, aunque tengan derecho de vivir en paz, «son qhinchas» (de mal agüero en aymara); sin embargo esto es algo que afecta a ellos mismos y no a los demás: «con mala suerte han nacido. Viven solitos, no tienen buena vida ino ve?». Nuevamente, se refiere al hecho de no poder conformar una unidad doméstica completa y no a la carencia económica, porque tanto el Gordo como Bernabé tienen suficientes ingresos.

\section{LA HOMOSEXUALIDAD FEMENINA}

A diferencia de los casos que hemos conocido de homosexuales varones, la mayoría de las homosexuales mujeres identificadas son o han sido casadas y tienen hijos. Aunque estén ya separadas de sus exmaridos, mantienen una unidad doméstica con sus hijos y/o hijas, y entre ellas figura el único caso que conocimos de una pareja homosexual que sí vivía abiertamente en sociedad conyugal. Se trata de una pareja que vivía en el pequeño pueblo de Qamaqi en los Yungas de Inquisivi. La mayor, Victoria, era de una familia de vecinos (clase media) del pueblo y era de vestido, y su conviviente, Felipa, era «una cholita» (de pollera) de otra comunidad de la provincia. El exmarido de Victoria, con quien tenía tres hijos varones, había desaparecido años antes de que Spedding la conociera en 1987 - la gente decía que había ido a vivir a la ciudad de Oruro y no se sabía nada de él en el pueblo- pero era de la familia más importante entre los vecinos. Una informante relataba que, en el pasado, cuando aún vivían juntos e iban a traer leña, Victoria rajaba troncos con hacha y luego subía las cargas al caballo, mientras 
Gemelón se limitaba a recoger y alistar las haces de leña rajada, «como mujer». Cuando Victoria vivía con Felipa, esta se encargaba de las tareas domésticas, mientras se veía a Victoria volviendo del trabajo agrícola en las tardes, machete en mano, caminando a grandes pasos, vestido de pantalón y malla y con su bolsa de coca amarrada a la cintura (y no llevado dentro de su aguayo como acostumbran las mujeres). Cuando no estaba trabajando y en ocasiones formales, sin embargo, usaba blusa y la falda estrecha entonces de moda entre las de vestido, aunque alguna vez bailó travestida como jilaqata (autoridad comunal, con poncho y chicote) en una comparsa de morenada en la fiesta del pueblo.

Debido a la evidente diferencia de clase entre las partes, un observador casual hubiera podido confundir su relación con un caso de relación entre patrona y empleada doméstica. Se confirmó que todos sabían que era más que eso cuando un primo de Victoria falleció en 1989. Durante el velorio, ella asumió el rol normalmente masculino de convocar a intervalos a los y las presentes a ponerse de pie y luego dirigir los rezos para el alma. Al día siguiente, cuando a las tres de la tarde se disponían a llevar al difunto al cementerio, ella no estaba presente y hacía falta su presencia. Al fin un vecino dijo «Mándale a llamar con su... con su... con su cholita, pues» y dieron el encargo a Felipa. Spedding supuso que esto indicaba una aceptación general de su relación. Se sorprendió cuando, al estar de paso en Qamaqi en 1991, encontró que ellas ya no estaban allí. La explicación dada fue «Mucho hablaban, "¿Qué es lo que hacen entre dos mujeres?" y se han separado». Pero poco tiempo después, yendo por un camino de herradura hacia dos otros pueblos del municipio, encontró a Victoria y a Felipa andando juntas en la dirección opuesta. Resultó que habían ido a vivir al campamento minero de Chilaya cerca del pueblo de Cajuata, operando una pensión donde comían los mineros. Su amor pudo más que las habladurías y duró hasta la muerte inesperada de Felipa unos años más tarde.

Rosa, que había vivido un tiempo en el mismo pueblo, aseveraba que Felipa no era la primera pareja conocida de Victoria. Tuvo otras antes de ella. «Y siempre tienen hijos, deben ser para ella ¿no?» A la fecha, Rosa estaba con su tercer marido (aunque solo se casó, por civil, con el segundo) y había llegado a tener un total de 12 hijos, de los cuales sobrevivían 10. De soltera, había trabajado de palliri (seleccionadora de mineral) en la mina de La Argentina, en la cordillera de Quimsa Cruz. Los fines de semana, ella y sus amigas compañeras de trabajo bajaban al pueblo de Quime a divertirse.

Y yo ponía una caja (de cerveza), y después mi amiga ponía una caja. Y después yo se le metía (la mano) a mi amiga, y después ella se la metía a mí.

No olvidaba sus relaciones lésbicas una vez iniciada su accidentada carrera conyugal, y sabía de la historia de Victoria porque ella había figurado entre sus amantes. Se desconoce si sus hijos (y maridos) sabían de su doble vida, aunque su hija mayor (heterosexual) expresaba una visión positiva de la posibilidad de la vida conjunta de una pareja de lesbianas, que podría ser una manera tácita de indicar que sí sabía y no estaba en contra. 
Ninguna de las mujeres de conocidas trayectorias lésbicas en Takipata llegó a vivir abiertamente en algún momento con una pareja del mismo sexo; las dos que se conocen en esta comunidad son o han sido casadas. La más activa, Francisca, se separó hace más de una década de su exmarido, con quien tuvo dos hijos. El conocimiento que él tenía de la doble vida de su entonces esposa salió a la luz en el curso de una disputa sobre la custodia del hijo menor en 2012. El exmarido llegó inesperadamente una noche y encontró al hijo solo en el balcón de la casa cerrada con llave y sin noticias de su madre. El chico empezó a llorar y le contó que no era la primera vez que le dejaba así, incluso sabía «encerrarse» con Matías, un casado de la comunidad, dejándole afuera. Cuando él había protestado le echó al suelo, pisándole el cuello y amenazando con matarle si se atrevía a hablar con alguien sobre lo acontecido. En realidad Matías era parte de un trío, cuyo tercer miembro, su esposa Paulina, siempre andaba de la mano con Francisca en el trabajo, en las fiestas y en todos los demás acontecimientos; se sentaban lado a lado y se echaban una en el regazo de la otra delante de todos.

Estos tratos afectuosos entre amigas, y también amigos, son habituales en las comunidades, y enteramente aceptables, como señala Ortiz Rescaniere, incluso si van más allá de las simples caricias, como era el caso entre estas dos. Durante la querella con el exmarido, Paulina fue a dormir al lado de Francisca para «acompañarla». La separacion de Francesca habia sido bastante conflictiva, motivada por el adulterio en flagrante de su marido; los demás comunarios aseveraron que hubo un acuerdo con Francisca para nunca recibirle más en la comunidad, y dar parte a los dirigentes para expulsarle si volviera. Aquel acuerdo fue incumplido por ella al haber permitido que llegue a su casa varias veces antes de la noche de marras. En el momento de presentar su denuncia y con fines de justificar su propia conducta, el exmarido aseguró que no solo él había sido infiel, sino también ella, no con hombres sino con mujeres: por ejemplo, con cierta profesora soltera nombrada en la escuela de la comunidad años atrás. Alguna mañana, él estaba caminando con su entonces esposa hacia el centro de la comunidad cuando ella insistió en desviar de la ruta normal para evitar pasar por delante de la vivienda de la profesora porque no quería que la viera en ese momento. El la presionó para que le contara cuál era el problema que la obligaba a evitar a la otra, y eventualmente ella soltó que la profesora era su amante y habían tenido una desavenencia amorosa.

Parte del acuerdo de separación fue que Francisca asumiera como afiliada titular en la lista del sindicato en lugar de su exmarido. Desde entonces ha pasado varios cargos: Secretaria de Vinculación Femenina (dos veces, la primera aún como casada), Secretaria de Vialidad, Secretaria de Agricultura (dos años, el segundo como ratificación) y Secretaria de Deportes (trocado por Vinculación Femenina otra vez porque este cargo había caído por los azares de la votación en un varón). Paulina no figura en la lista — su marido es el titular — pero ha sido catequista de la comunidad, luego fue postulada durante una elección sindical y salió en segundo lugar, siendo Secretaria de Relaciones en 2012. Posteriormente la comunidad la nombró candidata a la Federación Provincial de Trabajadores Campesinas de Sud 
Yungas-Bartolina Sisa y ganó, siendo Ejecutiva Provincial para la gestión 20132015. Su ejercicio no ha sido carente de observaciones, pero ninguna tiene que ver con las irregularidades de su vida personal reveladas en 2012. Según los comunarios, la única consecuencia de esto ha sido que ahora guarda total silencio frente a casos de mujeres acusadas de infidelidad conyugal, mientras que antes era una de las primeras en lanzar feroces críticas moralizantes. Su amiga íntima siempre está presente en las asambleas y demás actividades que ella preside como Ejecutiva. Es destacable que los maridos de ambas eran uxorilocales, es decir que los terrenos ocupados por sus unidades domésticas son herencia de las mujeres: en Yungas las mujeres heredan tierras con los mismos derechos que los varones. Las dos son de pollera, y aunque la Ejecutiva anda a veces con buzo (pantalón deportivo) en días ordinarios en la comunidad, muchas mujeres lo hacen en el trabajo agrícola aunque no sean formalmente de vestido (o «de pantalón» como se suele decir ahora en el campo) y no cuenta como travestismo. Así, tampoco el uso de ropa masculina por parte de Victoria contaría propiamente como tal, aunque su gestualidad cuando estaba así vestida, definitivamente le daba un aire varonil.

Estamos de acuerdo con Canessa que no hay una palabra aymara para «lesbiana» - q'iwsa, y su variante qiwa, solo refieren a hombres, y la urquchi es la mujer que no gusta de cocinar (y cocina mal cuando tiene que hacerlo) mientras realiza tareas varoniles con gusto, sin relación con su sexualidad-. Un informante de Takipata indicó que qiwa no significa homosexual, se refiere a una persona tacaña. A la vez sugirió que el nieto del exhacendado sería q'iwsa no por motivos sexuales, sino por ser «muy delicado» y nunca querer beber, es decir, emborracharse al máximo, lo que significa que carece de un comportamiento fundamentalmente varonil. Su evidente maternidad libera a las lesbianas de cualquier sospecha de anormalidad física. Varios hombres declaran que «para mí, una mujer sin hijos no es una mujer», pero tal mujer es denominada «una mula» (porque las mulas son estériles), sin tacharla de conducta sexual desviada. Podemos identificar un prejuicio patriarcal en la idea, también mencionada por Canessa, que el sexo entre mujeres simplemente no es sexo porque no hay penetración, y el hecho de haber incurrido en relaciones con alguien del mismo sexo no afecta la identidad de género de una lesbiana de la misma manera que ocurre con un varón homosexual, al menos cuando este es conocido por haber asumido el rol pasivo (penetrado). Rosa, la expalliri, se quejó alguna vez que en el curso de una relación lésbica «hace falta el clavo» (pene) y suplió la ausencia con la ayuda de una zanahoria larga y gruesa, pero no tenemos otros datos sobre el uso de juguetes sexuales parecidos (dildos o consoladores) en el área rural, aunque sí entre las mujeres internas en una cárcel femenina en la ciudad de La Paz.

A diferencia de los varones homosexuales, cuya sexualidad no solo les excluye de la convivencia abierta y permanente en pareja sino también de tener hijos7 - elemento fundamental de la unidad doméstica campesina, no solo como

7 Debido a que los varones considerados homosexuales asumen siempre el rol pasivo en el acto sexual. Puede haber «homosexuales» casados y con hijos, pero éstos asumirían el rol activo con cualquier pareja y por tanto no se los consideraría homosexuales. 
demostración de la sexualidad activa y fértil, sino como componente económico por su mano de obra impaga - pueden tener familia $y$, aunque ya sin marido (o quizás sin haberlo tenido nunca), formar una unidad doméstica con su prole que es una variante normal de la composición doméstica. Esta situación puede corresponder tanto a una separada o una viuda como a las «mujeres solas» que tienen hijos sin jamás haber convivido con sus padre(s), un tipo de familia matrifocal establecido desde hace tiempo en los Yungas, económicamente factible debido a la alta demanda de mano de obra femenina asalariada en la cosecha. Los ingresos permiten a estas mujeres contratar mano de obra masculina para las pocas tareas, como la plantación de coca o la construcción de casas, donde es imprescindible. En contraste, la mano de obra femenina doméstica (la cocina) solo circula, en el campo, a través del parentesco - no existen las empleadas domésticas- y por tanto, el hombre que no tiene madre o esposa o hija que le cocine, tiene que hacerlo él mismo lo mejor que pueda, de la misma manera que tiene que hacerse cargo de su propia cosecha de coca. Entonces, ser lesbiana no margina a estas mujeres de la misma manera que ser homosexual margina a los varones. Esto puede ser la raíz de la menor elaboración social y terminológica del lesbianismo, y no (o no solamente) el sesgo patriarcal que no quiere dar importancia a algo que, por definición, es solamente «entre mujeres».

\section{CONCLUSIONES}

Queda demostrado que, en las dos provincias yungueñas estudiadas aquí, los y las homosexuales no son discriminados en el acceso a la tierra, en la participación política ni en la vida social y ritual de la comunidad (por ejemplo, pueden pasar preste en una fiesta católica) ${ }^{8}$. Es lo contrario de lo encontrado, por ejemplo, por Ledezma Main (2005) que entrevistó a personas procedentes de diferentes comunidades del Altiplano. Encontró que hay un rechazo a la homosexualidad y que esto es más pronunciado entre las personas de mayor edad y más entre las mujeres que entre los hombres. Es posible que este resultado se deba al método utilizado, es decir una encuesta donde se piden opiniones generales: en el contexto artificial de una encuesta, las personas pueden preferir expresar

8 Hemos encontrado un ejemplo aislado de una valoración ritual positiva de la homosexualidad. Se trata de un rito llevado a cabo por un yatiri (curandero tradicional) de Takipata, para curar a un hombre de alcoholismo crónico, que ya había llegado al punto de arriesgar su vida si seguía tomando. El rito fue patrocinado por uno de sus hijos. El yatiri «sacó suerte», es decir que, para tener éxito, tenía que acompañarles en el rito cuatro personas (además de él, los familiares del paciente y el mismo paciente): dos hombres casados, un joven soltero y «uno que no le gusta las mujeres». El hijo que relató esto a Spedding, siendo lego en estos asuntos, no pudo indicar si los acompañantes tenían que desempeñar ciertos roles o actos rituales particulares aparte de simplemente presenciar el rito durante toda su duración. Por tanto falta mayor información para analizar el significado de convocar a un homosexual varón como elemento necesario para garantizar el éxito del rito (después de un segundo rito que no requirió esta vez de acompañantes, sí ha logrado alejar al paciente del trago, al menos según su hijo). Pero en este caso al menos, ser homosexual es todo lo contrario de qhincha. 
una opinión del «deber ser», demostrando actitudes de rectitud o según la moral convencional, que no corresponden a su conducta cotidiana. Es lo que pasó con el informante de Takipata que expresó que la homosexualidad es mala y hasta debe ser prohibida, pero nunca ha expresado rechazo personal por los homosexuales que conoce en su comunidad. Algo parecido puede explicar la condena de la homosexualidad expresada por algunos indianistas, cuya ideología tiende a idealizar el «mundo andino» como parte de los «pueblos indígenas» quienes, en palabras de Evo Morales, son «la reserva moral de la humanidad». No roban, no mienten, no son flojos, y tampoco deberían ser lesbianas ni maricones, y si contravienen esta moral, solo puede ser por haber caído bajo la influencia malvada del «Occidente». Nuestros hallazgos son la confirmación empírica del comentario, lamentablemente carente de contexto o fuentes, de Ortiz Rescaniere (1993), a la vez que la posición de este autor contrasta con las otras fuentes sobre homosexualidad rural que hemos consultado.

Los datos aportados por Spedding provienen de encuentros casuales con personas homosexuales en el curso del trabajo de campo cuyos temas centrales eran otros; nunca se puso a preguntar directamente sobre el tema. Cuando Vichevich lo hizo, aún contando con la amistad construida años atras con algunos de sus informantes, el más conocido de estos terminó indicando que era ofensivo ponerse a indagar directamente sobre la vida privada de las personas; además, insistir sobre el tema podría inducir a algunos a pensar que él mismo fuera homosexual (y estaría buscando pareja de manera disimulada). Varios informantes enfatizaron que no se puede objetar lo que la gente hace «en sus casas», es decir, fuera de la vista de los demás, al fin, siempre con discreción. Lo mismo se aplica al adulterio. En aymara tampoco goza de una palabra específica; designa el eufemismo jan wal sarnaqaña, «andar mal» en el castellano local, y todos están de acuerdo que es un acto inmoral, pero se conocen varias parejas de adúlteros, algunas que han mantenido sus relaciones durante décadas (y con la venia tácita de sus cónyuges) sin ser objeto de rechazo y menos castigo. Los que sí han sido objeto de sanciones (en algunos casos multas elevadas de dinero en beneficio del sindicato) son aquellos cuyas relaciones han Ilegado a ser de conocimiento público y escandaloso. Es el caso de la mujer separada y del hombre casado cuyas relaciones llegaron a ser filmadas en un video que luego pasó de celular en celular en la comunidad y hasta fue conocido por los hijos de los involucrados y otros escolares menores de edad, lo que fue el colmo de escándalo. Es relevante que el acto coital filmado haya ocurrido durante una borrachera en la casa del Gordo, y él hizo correr la noticia en forma verbal. Aunque no le atribuyeron la diseminación del video, propagar este chisme fue citado como prueba de ser él un chismoso sin remedio y, en ocasiones, dañino.

Las personas son identificadas con su comunidad y se considera que si corren noticias de actos inmorales o delictuosos (como por ejemplo, un robo) en, digamos, Takipata, el resto de la población va a comentar «En Takipata hacen esas cosas»y y de alguna manera todos sus habitantes caerán bajo sospecha de ser adúlteros, ladrones, etc. Se fomenta una conducta de hipocresía colectiva, o 
mejor dicho un pacto de silencio, sobre todo frente a cualquier persona ajena a la misma comunidad, pero también dentro de la misma, tratándose de personas que no deberían enterarse de ciertos hechos, sea por su supuesta inocencia (los menores de edad) y/o porque no podrían resistir al impulso de ir a contarlo fuera del lugar. En tanto que la homosexualidad es percibida como un hecho deleznable que mella la reputación comunal, también es silenciada. Si el investigador insiste sobre el tema, o obliga a responder sobre ello en un contexto artificial, lo más fácil es repudiarlo verbalmente, o por lo mucho admitir que se conoce, pero añadiendo que es algo que nunca pasa aquí, sino en otros lugares o entre otros grupos sociales. El rechazo a que dos personas del mismo sexo vivan conyugalmente de forma abierta puede deberse a esta hipocresía, porque haría evidente algo a lo cual tienen derecho, pero solo cuando lo disimulan en contextos públicos. Quizás las habladurías que condujeron a Victoria y Francisca a ir a vivir en el ambiente anónimo de un campamento minero llegaron a ser insoportables cuando su pantalla de ser patrona y empleada ya no convencía a nadie, mientras dos hombres difícilmente hubieran podido disponer de tal disfraz ni siquiera por poco tiempo. Cuando se enteró de este caso, la interpretación de Spedding era que lo reprochable no era su sexualidad, sino el hecho de haber conformado una unidad doméstica entre dos mujeres donde una hacía «de hombre» y la otra «de mujer», según la división del trabajo por género. La organización de la unidad doméstica como célula productiva de la economía campesina se fundamenta en esta distribución complementaria de las tareas. Esto obliga a la gran mayoría de los y las habitantes de las comunidades, primero - temprano o tarde- a «juntarse» con una pareja, que se supone de antemano será del sexo opuesto, y luego a mantenerse junto con esta pareja en lo laboral y doméstico (aunque hayan dejado de tener sexo, o estén teniéndolo con otra persona). Cuando la separación resulta inevitable no obstante la presión social de seguir juntos, deben tratar de buscar un reemplazante, de ser posible (los viudos y viudas, en lo ideal, deberían respetar tres años de duelo nominal antes de tomar una nueva pareja; no hay reglas para separados y separadas). Como hemos explicado, es más factible para una mujer sola que para un hombre encabezar una unidad doméstica; ellas no necesitan a un hombre tanto como éstos las necesitan a ellas ${ }^{9}$, y por esto las viudas y separadas no se afanan tanto en buscar un segundo cónyuge como los viudos, mientras algunas mujeres optan por ser madres cabeza de familia sin tener nunca marido. Entonces, la heterosexualidad obligatoria $-\mathrm{o}$ formal, como en el caso de las lesbianas casadas - beneficia más a los hombres que a las mujeres. Por eso, ser homosexual y tener que renunciar a esta posibilidad es visto como un destino desafortunado para un varón, mientras ningún informante se compadecía de las lesbianas. Spedding pensó que el rechazo a Victoria y Francisca expresaba tácitamente que ellas eran una especie de «mal ejemplo» para las demás mujeres, porque ponían en evidencia que era posible tener una buena vida sin tener que servir a un hombre, e incluso sin tener que manejar la unidad doméstica

9 Este tema es desarrollado en mayor extensión en Spedding (1997). 
sola — haciendo las tareas de dos o erogando dinero para contratar la mano de obra necesaria para cubrir las tareas que el marido hubiera hecho impago-. La fuerza del modelo de complementariedad de género es tal que se supone que, en caso de establecer una unidad doméstica entre dos varones, uno de ellos asumiría «de mujer» y el otro «de hombre». El mayor prestigio simbólico acarreado por los roles considerados varoniles hace admirable a estas mujeres que se disponen a realizarlos, mientras que el hombre que asume roles de mujer no gana prestigio por su capacidad múltiple: apenas un mínimo de respeto para enfrentar su desgracia (el solterón u hombre abandonado por su mujer que tiene que cocinar para él mismo) y cierta sorna cuando esto es visto como una decisión voluntaria (como estos jóvenes que se burlaron del Gordo después de que él les haya contratado para realizar un trabajo «de hombres» que él no quiso o pudo cubrir por sí mismo). Es notable que «saber cocinar», es decir dominar una tarea emblemática de las mujeres, es atribuido a «todos los maricones» como si fuera consustancial a su opción sexual, mientras que no se considera ninguna actividad varonil como típica de las lesbianas. El hecho de que algunos homosexuales varones optan por dejar sus comunidades e ir a las ciudades, puede deberse no tanto al rechazo directo sino a las facilidades para comprar servicios domésticos (comer en pensiones, pagar a una lavandera) en el área urbana.

La opción de pagar mano de obra masculina está abierta para las mujeres yungueñas por su amplio acceso al trabajo asalariado (jornalear en la cosecha de coca) mientras en muchas regiones altiplánicas, hay poco trabajo asalariado disponible y cuando existe, suele ser mayormente para varones. Esto crea mayor necesidad de un varón «propio» en casa para las mujeres, e induce a soportar las conductas más brutales de dominación masculina, dominación cuestionada simbólicamente por los q'iwsa, quienes aceptan actuar «como mujeres» en el trabajo y en la cama. Este hecho origina anécdotas que se cuentan como la de un campesino del norte de Potosí que se preocupó por curar su vaca pero no así a su mujer, alegando que si ella moría, fácilmente iba a conseguir otra en su lugar, mientras sería costoso reemplazar a la vaca —actitud que no se observa en Yungas, donde, aparte de no tener vacas, la enfermedad discapacitante o muerte de la esposa deja al esposo en serios apuros para conseguir cosechadoras-. Sin una cosecha oportuna, su coca se malogra y queda sin ingresos, o sino ve reducido sus ingresos al tener que pagar mingas en vez de conseguir ayni con la ayuda de su mujer, mientras que se supone que no hay tantas mujeres listas para entregarse a cualquier hombre (como por ejemplo un viudo con hijos quienes no van a querer de entrada a una madrastra). Si fuera cierto que en el Altiplano las mujeres están más en contra de la homosexualidad (masculina, tratándose la categoría marcada en este tema) que los hombres iexpresarían un rechazo de estos varones que voluntariamente se hacen no disponibles para ellas, reduciendo aún más la posibilidad de tener un marido-trabajador en un contexto donde la migración estacional de los hombres ha provocado una escasez de mano de obra masculina? Pero esto es entrar en la especulación; se requieren más investigaciones de campo para averiguar sobre las prácticas y las actitudes hacia la homosexualidad en otras regiones de los Andes antes de lanzarse a conclusiones generales sobre el tema. 


\section{Referencias citadas}

CANESSA, A., 2006 - Minas, mote y muñecas. Identidades e indigeneidades en Larecaja, 217 pp.; La Paz: Mama Huaco.

HURTADO, E., 2011 - Ser gay en tiempos de Evo, 145 pp.; Sin lugar de edición: Ayni.

HURTADO, E., 2015 - La Madonna de Sorata. Crónicas sobre indígenas homosexuales en Bolivia, 132 pp.; Sin lugar de edición: Ayni. Segunda edición.

LEDEZMA MAIN, J. K., 2005 - Percepción del aymara boliviano sobre la homosexualidad. In: Actas de la Reunión Anual de Etnología 2004; La Paz: MUSEF.

ORTIZ RESCANIERE, A., 1993 - La pareja y el mito. Estudio sobre las concepciones de la persona y de la pareja en los Andes, 261 pp.; Lima: Fondo Editorial de la Pontificia Universidad Católica del Perú. Segunda edición.

RIVERA, S., 2013 - Homosexualidad sagrada, tradiciones inventadas y el discurso del chacha-warmi. In: Reflexiones a las diversidades sexuales y de género en comunidades indígenas de Bolivia (Vargas \& Aruquipa, eds.); La Paz: Comunidad Diversidad.

SPEDDING, A., 1997 - "Esa mujer no necesita hombre": en contra de la "dualidad andina" - imágenes de género en los Yungas de La Paz. In: Más allá del silencio: las fronteras del género en los Andes (D. Arnold, ed.): 325-343; La Paz. CIASE/ILCA.

SPEDDING, A. \& FLORES, G., 2014 - ¿Refugios fuera del poder o poderes alternativos? El comercio y las fiestas en Chulumani (Sud Yungas, La Paz). Temas sociales, 35: 45-78; La Paz.

VARGAS, P. \& ARUQUIPA, D. (eds), 2013 - Reflexiones sobre diversidades sexuales y de género en comunidades indígenas de Bolivia, 47 pp.; La Paz: Diakonia. Comunidad Diversidad, año 1, n. $^{\circ} 1$. 